\title{
Cold dust in (some) high-z supernova host galaxies
}

\author{
D. L. Clements, ${ }^{1 \star}$ D. Farrah, ${ }^{2}$ M. Rowan-Robinson, ${ }^{1}$ J. Afonso, ${ }^{3}$ R. Priddey ${ }^{4}$ \\ and M. Fox ${ }^{1}$ \\ ${ }^{1}$ Imperial College, London, Blackett Lab, Prince Consort Road, London SW7 2BW \\ ${ }^{2}$ Spitzer Science Centre, Jet Propulsion Laboratory, California Institute of Technology, Pasadena, CA 91125, USA \\ ${ }^{3}$ CAAUL, Universidade de Lisboa, Faculdade de Ciências, Observatório Astronómico de Lisboa, Tapada da Ajuda, 1349-018 Lisboa, Portugal \\ ${ }^{4}$ Department of Physics, Astronomy and Mathematics, University of Hertfordshire, Hatfield, Herts AL10 9AB
}

Accepted 2005 July 14. Received 2005 July 11; in original form 2005 June 7

\begin{abstract}
We present deep submillimetre photometry for 14 galaxies at $z=0.5$ that are hosts of Type Ia supernovae, with the aim of examining the evolution of dust mass and extinction in normal galaxies. We combine these results with our previous observations of $17 z \sim 0.5 \mathrm{SN} 1 \mathrm{a}$ hosts to look for any evolution in the dust content of normal galaxies between $z=0$ and 0.5 . The average observed-frame $850-\mu \mathrm{m}$ flux of SN1a hosts in the full sample, excluding two bright individually detected objects, is $0.44 \pm 0.22 \mathrm{mJy}$. This flux level is consistent with there being little or no evolution in the dust content, or optical extinction, of normal galaxies from $z=0$ to 0.5. One galaxy, the host of SN1996cf, is detected individually, and we also present a deep Hubble Space Telescope (HST) Space Telescope Imaging Spectrograph (STIS) image for this object. It appears to be an edge-on disc system, similar to the submillimetre bright host of SN1997ey. We thus examine the dust properties of these and one other individually detected object. Flux ratios and limits of 450-850 $\mu \mathrm{m}$ suggest that the dust in the two brightest submillimetre sources, SN1996cf and SN1997ey, is cold, $T \sim 20 \mathrm{~K}$, implying that they contain a substantial mass of dust $\sim 10^{9} \mathrm{M}_{\odot}$. The presence of two bright $\left(F_{850}>7 \mathrm{mJy}\right)$ submillimetre sources at $z \sim 0.5$ in a sample of ostensibly normal galaxies is surprising, and has important implications. It supports the idea that a substantial part of the cosmic infrared background (CIB) may be produced at $z<1$, while also suggesting that 'foreground' objects such as these may be a significant 'contaminant' in submillimetre surveys. Finally, we examine the overall submillimetre luminosity distribution at $z=0.5$ implied by our results, and conclude that either there is substantial evolution in the submillimetre luminosity function from $z=0$ to 0.5 , or our submillimetre-detected sources are somehow not representative of the bulk of galaxies at this redshift.
\end{abstract}

Key words: supernovae: general - galaxies: high-redshift - submillimetre.

\section{INTRODUCTION}

Two of the major new insights of the 1990s into cosmology and the history of galaxy formation were the discovery of the cosmic infrared background (CIB; Puget et al. 1996; Fixsen et al. 1998) and the measurement of a significant 'dark energy' term in the expansion of the Universe through the use of high-redshift Type Ia supernovae ( $\mathrm{SNe}$ ) as standard candles (Reiss et al. 1998, HZT; Perlmutter et al. 1999, SCP). The discovery of the CIB indicated that dust-enshrouded star formation is a significant aspect of the star formation history of the Universe, amounting to 50 per cent or more of all star formation (Gispert, Lagache \& Puget 2000). At the

^E-mail: d.clements@ic.ac.uk same time, surveys with the Submillimetre Common-User Bolometer Array (SCUBA) (e.g. Smail, Ivison \& Blain 1997; Hughes et al. 1998; Eales et al. 2000; Mortier et al. 2005) and other submillimetre array detectors had begun to find the objects that make up the CIB. These objects are largely interpreted as being similar to local ultraluminous infrared galaxies (ULIRGs), and are thought to contain large dust masses at a temperature $T \sim 40 \mathrm{~K}$ (Blain et al. 1999) and to be the hosts of massive bursts of star formation, $100-1000 \mathrm{M}_{\odot} \mathrm{yr}^{-1}$. However, substantial uncertainties remain. Most of these submillimetre galaxies (SMGs) do not have well-determined redshifts (see Chapman et al. 2005). The degeneracy between temperature and redshift (Blain 1999) thus means that their dust temperature is highly uncertain - a low dust temperature object at low redshift $(z \sim 0.5-1)$ looks much the same as a high dust temperature object at high redshift $(z \sim 2-3)$. Since luminosity is a strong power of temperature, 
typically $T^{6}$ for a standard SMG spectral energy distribution (SED), this leads to a considerable uncertainty in the derived luminosity and star formation rates for those SMGs without measured redshifts. Indeed some authors have suggested (Rowan-Robinson 2001; Efstathiou \& Rowan-Robinson 2003; Kaviani, Haenelt \& Kauffman 2003) that a substantial fraction of the SMG population may be cooler and closer than originally thought, and there is some observational evidence to back up this suggestion (Chapman et al. 2003a; Taylor et al.). Currently, little is known about cool dust in normal galaxies in the range $0.5<z<1$ because it is quite difficult to find a clean sample of such objects. The SN1a host galaxies (see e.g. Sullivan et al. 2003; Tonry et al. 2003), though, provide an ideal selection for such studies.

The discovery of the CIB also raises the overall question of the evolution of the dust content of galaxies and its role in obscuring starlight in these objects and reprocessing it into thermal dust emission. Dust obscuration is known to have a significant effect locally (Tresse \& Maddox 1998), but the evolution of dust content and obscuration is not well constrained. Chemical evolution models (Calzetti \& Heckman 1999; Pei, Fall \& Hauser 1999) predict that dust obscuration should peak at two to three times the current value at $1<z<2$ before gradually declining to higher redshifts. However, we currently know little about the dust content of quiescent systems that make up the bulk of the galaxy population at any epoch. The host galaxies of high- $z$ SNe, however, have not been selected on the basis of active star formation, but have spectroscopic redshifts and abundant ancillary data, including, for many, Hubble Space Telescope (HST) images and multicolour optical and near-infrared colours. They are thus an ideal sample with which to study the role of dust in quiescent galaxies at cosmological redshifts.

This paper is the second to result from our ongoing search for dust emission in high- $z$ SN host galaxies. Our first paper (Farrah et al. 2004, hereafter Paper 1) presented the results of SCUBA submillimetre photometry of a sample of $17 z=0.5$ SN1a host galaxies. Here, we present additional observations of a further 14 galaxies, extending the sample size to 31 , and increasing the number of directly detected objects to 3 . The paper is structured as follows. In Section 2, we discuss the observations and present our results. In Section 3, we discuss the sample as a whole and discuss the overall dust content of the quiescent galaxy population at $z=0.5$. In Section 4, we examine the properties of the three galaxies individually detected in our observations, while in Section 5, we discuss the implications of our results for the overall submillimetre luminosity distribution of galaxies at $z=0.5$. We draw our conclusions in Section 6. We assume $\Omega_{0}=1, \Omega_{m}=0.3, \Omega_{\Lambda}=0.7$ and $H_{0}=$ $70 \mathrm{~km} \mathrm{~s}^{-1} \mathrm{Mpc}^{-1}$ throughout this paper.

\section{OBSERVATIONS}

The observations described here extend the earlier work of Paper 1 on the $z \sim 0.5$ Type Ia SN host galaxies. As with our Paper 1 observations, our targets were selected from the HZT and the SCP $\mathrm{SN}$ lists to be the host galaxies of Type Ia SNe and to lie in a narrow range $( \pm 0.1)$ of redshifts centred on $z=0.5$. This is to allow an easy combination of submillimetre fluxes from the targets with no need for $k$-corrections to shift the fluxes to the corresponding rest-frame wavelength.

Observations were made of 14 high-redshift SN1a host galaxies at $z \sim 0.5$ between 2003 July and 2004 January (see Table 1). Observing conditions were generally good, with $\tau_{850}$ ranging from 0.2
Table 1. Results of submillimetre observations of SN1a host galaxies.

\begin{tabular}{|c|c|c|c|}
\hline Name & $z$ & Date observed & $F_{850}(\mathrm{mJy})$ \\
\hline SN1995az & 0.45 & 2003 July 9 & $1.4 \pm 0.9$ \\
\hline SN1997ax & 0.615 & 2003 Dec 26 & $1.8 \pm 0.8$ \\
\hline SN1997K & 0.592 & 2003 Dec 16, 2004 Jan 9 & $-0.3 \pm 0.9$ \\
\hline SN1997H & 0.526 & 8, 2004 Jan 10 & $0.7 \pm 0.9$ \\
\hline SN1997eq & 0.538 & 2004 Jan 8 & $1.5 \pm 1.2$ \\
\hline SN1997j & 0.619 & 2004 Jan 8 & $0.4 \pm 0.9$ \\
\hline SN1997af & 0.579 & 9, 2004 Jan 10 & $0.0 \pm 0.8$ \\
\hline SN2000ec & 0.47 & 2004 Jan 10 & $-2.7 \pm 1.4$ \\
\hline SN1996au & 0.52 & 2004 Jan 10 & $-3.0 \pm 1.4$ \\
\hline SN1997es & 0.65 & 2004 Jan 10 & $-0.7 \pm 1.4$ \\
\hline $\mathrm{SN} 1996 \mathrm{cf}^{a}$ & 0.57 & 2004 Jan 10 & $11.0 \pm 1.6$ \\
\hline SN1996ci & 0.5 & 11, 2004 Jan 12 & $-0.7 \pm 0.8$ \\
\hline SN1997aj & 0.581 & 2004 Jan 12 & $-1.8 \pm 1.3$ \\
\hline SN1996I & 0.57 & 2004 Jan 12 & $-0.6 \pm 1.2$ \\
\hline
\end{tabular}

${ }^{a}$ SN1996cf $450-\mu \mathrm{m}$ flux $25 \pm 15 \mathrm{mJy}$.

to 0.15 and $\tau_{450}$ ranging from 1.3 to 0.85 . These optical depth values were determined by standard skydip observations at azimuths relevant to the targets, and are classified as grade 1 or 2 conditions by the James Clerk Maxwell Telescope (JCMT).

The sources were observed with SCUBA (Holland et al. 1999) in photometer mode using two-bolometer chopping. This means that for each channel, 850 or $450 \mu \mathrm{m}$, as well as being observed by the central bolometer on the array, a second bolometer observes the source in the reference position. An observed flux can be extracted from both these bolometers which are then combined, using appropriate noise weighting, to increase the sensitivity over that of a normal single bolometer chopping observation by $\sim \sqrt{2}$. This combination takes place at the end of the data reduction process, once flat fielding, background subtraction and calibration have been completed.

The data from SCUBA were reduced in a standard way using the SURF data reduction package (Jenness \& Lightfoot 2000). Regular pointing observations were made to ensure pointing accuracy, and skydips were also taken regularly. Flux calibration factors (FCFs) for the two bolometers used to detect the sources were calculated separately. Calibration sources used were CRL618 and Uranus. The individual observations of a source with a given bolometer are combined using a Kolmogorov-Smirnoff technique, which masks out any discordant points that remain after the data processing. No unusual numbers of points were excluded in this analysis. When an object was observed on more than one night, the fluxes measured in each separate observation are combined using a variance-weighted scheme.

Our results are presented in Table 1. Only one source, the host galaxy of SN1996cf, $z=0.57$ SN1a, was detected, with a flux of $11 \pm 1.6 \mathrm{mJy}$ at $850 \mu \mathrm{m}$. This source was only 'detected' with $1.6 \sigma$ significance at $450 \mu \mathrm{m}$, with a flux of $25 \pm 15 \mathrm{mJy}$.

For the host galaxy of SN1996cf, we obtained HST imaging from the HST data archive. Observations were taken using the Space Telescope Imaging Spectrograph (STIS) in imaging mode using a clear filter. The data consisted of three exposures, each of $300 \mathrm{~s}$, taken with a 10-pixel offset between successive exposures to facilitate the subtraction of cosmic ray events. The data were reduced, and combined into a single image, using the IRAF reduction package CALSTIS. The image of the host galaxy, presented in Fig. 1, has had the low surface brightness host enhanced by the application of a $2 \times 2$ pixel boxcar filter. 


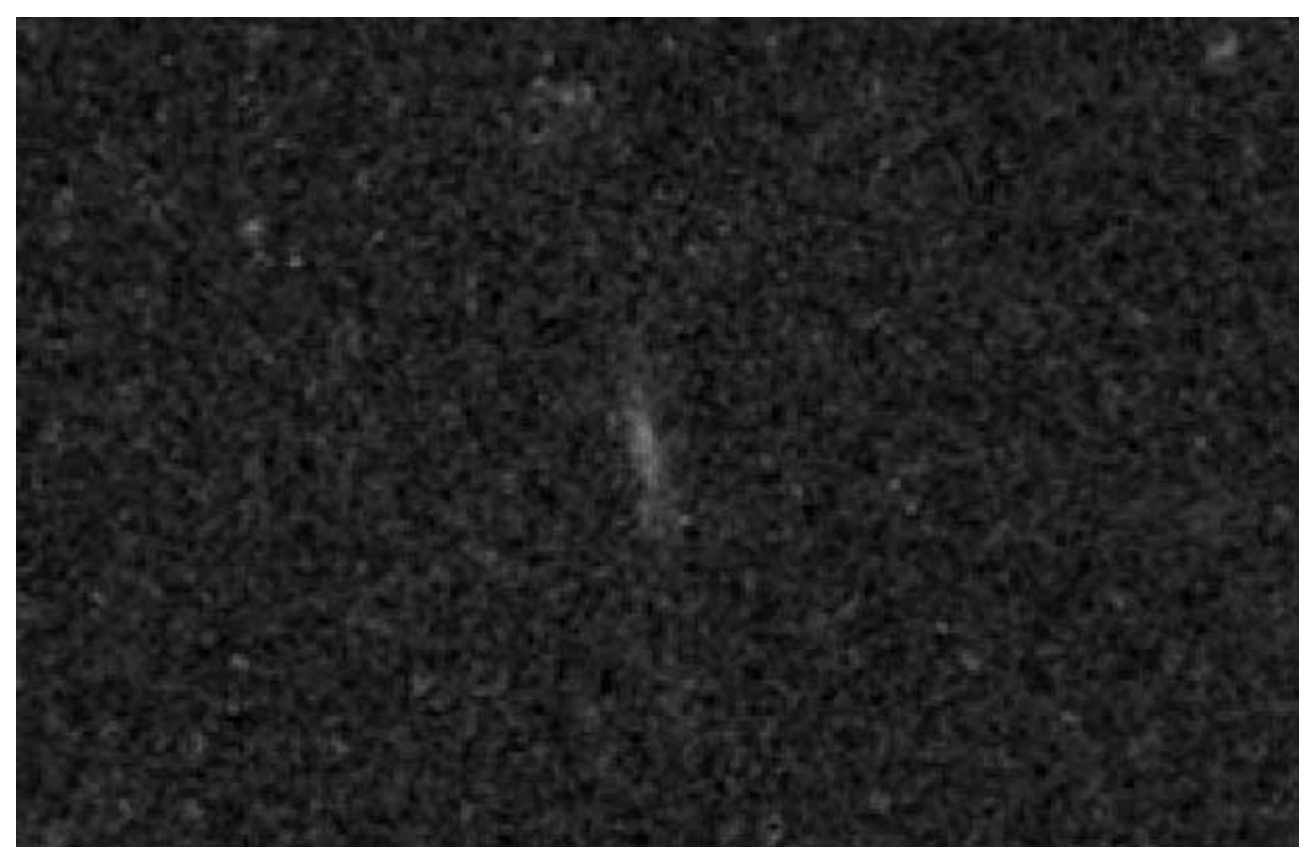

Figure 1. Space Telescope Imaging Spectrograph (STIS) clear CCD image of the host galaxy of SN1996cf. The pixel size is the standard STIS CCD resolution of $0.05 \mathrm{arcsec}$. The image is $15 \times 11 \mathrm{arcsec}$ in size, i.e. $300 \times 220$ pixel.

\section{EVOLUTION OF OBSCURATION IN NORMAL GALAXIES}

The evolution of dust content and dust obscuration in normal galaxies can be examined by looking at the average properties of the sample we have observed. We follow the approach discussed in Paper 1, but apply it to the full sample of 31 galaxies - 17 from Paper 1 and 14 from the current paper. We exclude two objects from this combination, the two objects whose fluxes are strongly detected - SN1996cf (this paper) and SN1997ey (Paper 1). The fluxes are combined using an inverse variance-weighting scheme. The average observed-frame 850- $\mu \mathrm{m}$ flux from Type Ia SN host galaxies calculated in this way is $0.44 \pm 0.22 \mathrm{mJy}$. This is less than the mean flux of $1.01 \pm 0.33$ mJy obtained in Paper 1, but still statistically consistent (at the $1.5 \sigma$ level) with the previous result. If we attempt to split the SN host galaxy sample into spirals (and irregulars) and ellipticals, based on the available data on the host galaxy morphologies (largely HST images), whilst still excluding the two strong detections, we find a similar average flux for the two classes of object $(0.40 \pm 0.29$ for spirals/irregulars and $0.69 \pm 0.46$ for ellipticals).

By consideration of the SCUBA Local Universe Galaxy Survey (SLUGS) 850- $\mu \mathrm{m}$ luminosity function (LF) (Dunne et al. 2000, see also Vlahakis, Dunne \& Eales 2005) combined with appropriate $k$-corrections from the cirrus model of Efstathiou \& RowanRobinson (2003), and the assumption that the submillimetre emission from ellipticals is negligible, Paper 1 predicted that the mean observed-frame $850-\mu \mathrm{m}$ flux of a $z=0.5$ galaxy would be $0.56 \pm$ $0.1 \mathrm{mJy}$ if there is no evolution in the amount of dust in normal galaxies from $z=0$ to 0.5 . Our result is thus consistent with there being at most only moderate evolution in the dust content of normal galaxies from $z=0$ to 0.5 . Paper 1 found a moderate increase in the dust content of 25-135 per cent over this redshift range. The new expanded $\mathrm{SN}$ host sample thus mildly contradicts this result $(1.5 \sigma)$, though the errors permit an increase of up to 20 per cent or a 100 per cent decrease in the dust content of the population as a whole. The mean $A_{V}$ for galaxies at $z=0.5$ would thus be statistically unchanged from the local value of $A_{V}=0.31$, derived by Rowan-Robinson (2003). This is in contrast with models of opacity evolution from Calzetti \& Heckman (1999), which predict an increase in extinction of $A_{V} \sim 0.15$ by $z=0.5$, and with chemical evolution models (Pei et al. 1999). Neither of these models predict a particularly significant evolution in extinction or dust mass in galaxies over the period to $z=0.5$, so our current results cannot be interpreted as a rigorous test of these models. To achieve this, a larger sample and more sensitive observations would be needed, allowing more than statistical non-detections of the population, and/or the greater lever arm that would be obtained by examining the dust content of normal galaxies out to $z=1$.

All of these considerations apply to the population as a whole. Throughout this discussion we have excluded the two strong submillimetre detections in our sample from consideration because the two objects appear to have radically different submillimetre luminosity to the bulk of the population. We now consider the nature of these two objects in detail.

\section{PROPERTIES OF THE DETECTED GALAXIES}

Whilst we have failed to detect the mean submillimetre emission of the population of $z \sim 0.5 \mathrm{SN} 1 \mathrm{a}$ host galaxies, we have managed to detect three of these objects individually. The SN magnitudes for these objects lie in typical positions for $z \sim 0.5 \mathrm{SN} 1 \mathrm{a}$ objects in the magnitude-redshift diagram (e.g. Perlmutter et al. 1999). One of our sources, SN2000eh, was only marginally detected $(3 \sigma)$ at $850 \mu \mathrm{m}$, but two others, SN1997ey and SN1996cf, were detected surprisingly strongly. Their observational and derived properties are given in Table 2 . It can be clearly seen that their rest-frame $850-\mu \mathrm{m}$ luminosities are comparable with those of local ULIRGs such as Arp220 and Mrk231 (Dunne et al. 2000; Farrah et al. 2003). However, there is no evidence for starburst or active galactic nucleus (AGN) activity in these objects on the basis of optical spectra obtained by the 
Table 2. Detailed observational properties of the strongly detected sources. For comparison, Arp220, the nearest ULIRG, and Mrk231, the nearest Type I AGN ULIRG, have 850- $\mu \mathrm{m}$ luminosities of $4.0 \times 10^{22} \mathrm{~W} \mathrm{~m}^{-2} \mathrm{sr}^{-1}$ and $1.0 \times 10^{23} \mathrm{~W} \mathrm{~m}^{-2} \mathrm{sr}^{-1}$, respectively.

\begin{tabular}{ccccccc}
\hline Name & RA & Dec. & $z$ & $F_{450}(\mathrm{mJy})$ & $F_{850}(\mathrm{mJy})$ & $L_{850}\left(\mathrm{~W} \mathrm{~m}^{-2} \mathrm{sr}^{-1}\right)$ \\
\hline SN1997ey & $04^{\mathrm{h}} 56^{\mathrm{m}} 58^{\mathrm{s}} .2$ & $-02^{\circ} 37^{\prime} 37^{\prime \prime}$ & 0.58 & $20.8 \pm 3.5$ & $7.8 \pm 1.1$ & $8.7 \times 10^{22}$ \\
SN1996cf & $10^{\mathrm{h}} 48^{\mathrm{m}} 50^{\mathrm{s}} .9$ & $00^{\circ} 03^{\prime} 30^{\prime \prime}$ & 0.57 & $25 \pm 15$ & $11.0 \pm 1.6$ & $1.2 \times 10^{23}$ \\
SN2000eh & $04^{\mathrm{h}} 15^{\mathrm{m}} 02^{\mathrm{s}} .4$ & $04^{\circ} 23^{\prime} 18^{\prime \prime}$ & 0.49 & $5.2 \pm 6.6$ & $4.6 \pm 1.5$ & $4.7 \times 10^{22}$ \\
\hline
\end{tabular}

high-redshift SN teams (HZT \& SCP teams, private communication). The HST imaging of the two bright submillimetre sources also shows them to be morphologically different from local ULIRGs and from those SMGs for which imaging is currently available. Both of our bright submillimetre sources (see Fig. 1 for 1996cf and Paper 1 for 1997ey) appear to be faint quiescent edge-on disc galaxies. Local ULIRGs, in contrast, are almost universally disturbed systems (e.g. Clements et al. 1996; Surace et al. 1998; Farrah et al. 2001), with double nuclei, tidal tails and other signs of merging activity. The SMG population, as revealed by blank-field SCUBA surveys, also appears to be made up of such disturbed objects (e.g. Chapman et al. 2003b; Clements et al. 2004; Smail et al. 2004), though it should be noted that to date no flux-limited SMG sample has been completely imaged.

The spectral energy distributions and dust temperatures of these objects are difficult to determine since they are detected in only one or two submillimetre bands. The best we can attempt is to set constraints on the temperature and SEDs by examining the 450/850 flux ratio and its limits. These targets were all observed in excellent conditions, so calibration errors should be smaller than or comparable to the observational errors on the fluxes (see e.g. Dunne \& Eales 2001). One source is clearly detected at $450 \mu \mathrm{m}, \mathrm{SN} 1997 \mathrm{ey}$, while a second, $1996 \mathrm{cf}$, has a marginal $1.6 \sigma$ signal. There are no useful $450-\mu \mathrm{m}$ data available for SN2000eh since it was observed in poorer conditions. Examination of the 450/850 flux ratio for 1997ey and 1996cf (see Fig. 2) suggests cold dust temperatures of $\sim 20 \mathrm{~K}$, assuming an emissivity index of $\beta=1.3$ (consistent with the single-temperature SEDs found for local galaxies by SLUGS (Dunne et al. 2000) and still colder for the more generally accepted emissivity index $\beta=2$ (see e.g. Dunne \& Eales 2001). Assuming a standard dust opacity of $0.077 \mathrm{~m}^{2} \mathrm{~kg}^{-1}$ (e.g. Dunne et al. 2000), these would correspond to dust masses of $1.3 \times 10^{9}$ and $1.7 \times$ $10^{9} \mathrm{M}_{\odot}$, respectively. In the absence of $450-\mu \mathrm{m}$ data, we assume that SN2000eh has similar dust properties, yielding a lower dust mass of $6.5 \times 10^{8} \mathrm{M}_{\odot}$. These are substantial dust masses, with about a factor of 10 more dust than in the dustiest galaxy in the original SLUGS, UGC9618 (Dunne et al. 2000). The low inferred temperature, combined with the large observed $850-\mu \mathrm{m}$ flux, lead inevitably to this large dust mass. If the cooler temperature associated with a $\beta=2$ SED is assumed, then the dust masses get correspondingly larger.

Dust as cold as we have found in these objects is not unknown in the local Universe (e.g. the SED of NGC891 is dominated by dust at $\sim 15 \mathrm{~K}$, Alton et al. 1998) and has been suggested for higher
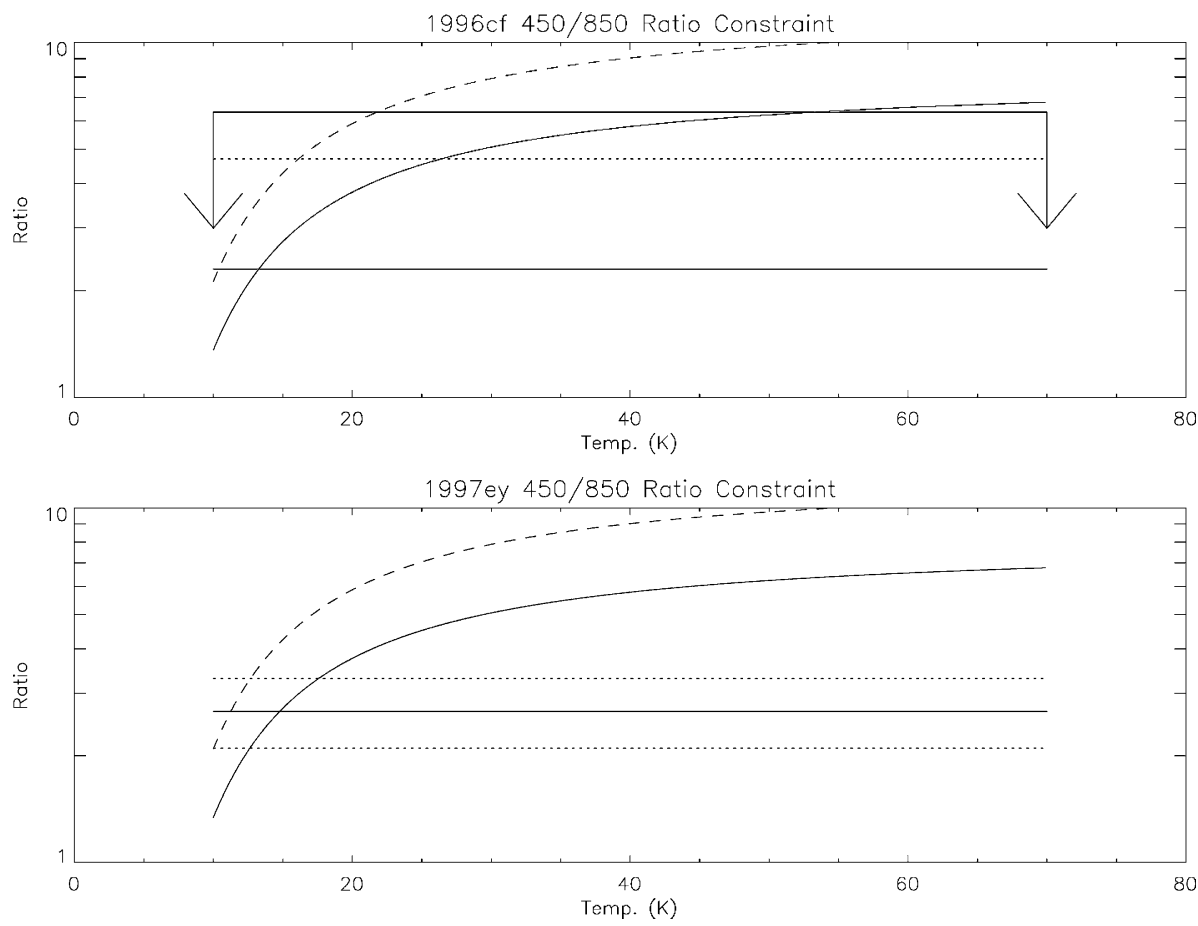

Figure 2. Comparison of dust SED models to the $450 / 850$ ratio of strongly detected sources. The curved lines are the predicted observed-frame $450 / 850$ ratios for thermal dust emission models with a $\beta=1.3$ emissivity (dashed line) or $\beta=2$ emissivity (solid line). The horizontal lines are the observed ratios. Solid lines show the observed values and, for SN1996cf, the upper limit to this ratio using the formal 450- $\mu \mathrm{m} 3 \sigma$ flux upper limit (designated with an arrow), while dashed lies show the $1 \sigma$ range for this flux ratio. As can be seen, SN1997ey appears to have a cold SED, with a temperature of $20 \mathrm{~K}$ or less, while it seems likely that SN1996cf's host is similar. 
redshift objects on both theoretical grounds (Rowan-Robinson 2001; Efstathiou \& Rowan-Robinson 2003; Kaviani et al. 2003) and observational grounds (Chapman et al. 2003a; Taylor et al. 2005). The present results are direct confirmation that cold galaxies with large dust masses and submillimetre luminosities do indeed exist at moderate redshifts, and could contribute to the CIB and the sources seen in deep far-infrared (FIR) surveys such as FIRBACK (Puget et al. 1999) and deep submillimetre surveys. Indeed, objects such as these might prove to be a foreground contaminant in deep large-area submillimetre surveys such as SHADES (SCUBA HalfA-Degree Extragalactic Survey; Mortier et al. 2005) and those now being planned for new facilities and instruments such as SCUBA2 and the LMT (Large Millimetre Telescope). The areal density for sources such as the two bright galaxies discussed here was predicted in Paper 1 to be $\sim 100 \mathrm{deg}^{-2}$. The results of this paper, which roughly doubles the sample size and doubles the number of bright sources found, add credence to this number, which is comparable to the source densities found in deep blank-field submillimetre surveys at these flux levels (e.g. Scott et al. 2002; Mortier et al. 2005). The presence of such foreground interlopers might thus be a significant problem when attempting to measure correlation functions for highredshift SMGs in the absence of clear identifications and redshifts, especially as they are unlikely to be bright in the radio.

\section{$5 z=0.5$ SUBMILLIMETRE LUMINOSITY DISTRIBUTION}

In principle, our sample of SN1a host galaxies should provide a reasonably unbiased selection for evolved galaxies at $z=0.5$, biased in selection only by the stellar mass. By examining the $850-\mu \mathrm{m}$ luminosity distribution of these objects, and comparing it with that found for local objects, we can gain some insight into the galaxy population at $z=0.5$. The local LF that we use is the SLUGS $850-\mu \mathrm{m}$ LF (Dunne et al. 2000) since this is the best currently available. This was found to be well fitted by a Schechter function with $L_{*}=8.3 \times$ $10^{21} \mathrm{~W} \mathrm{~m}^{-2} \mathrm{sr}^{-1}, \alpha=-2.18$ and $\Phi_{*}=2.9 \times 10^{-4} \mathrm{Mpc}^{-3} \mathrm{dex}^{-1}$. We cannot apply this LF directly to predict the numbers of sources we should see since we do not know the parent population normalization for the number of our target objects per $\mathrm{Mpc}^{-3}$. However, since all our objects are at the same redshift, $\sim 0.5$, we can look at the relative number of objects in each luminosity bin and compare this to the low- $z$ expectation. We have only a small number of detected galaxies, so must necessarily normalize the expected luminosity distribution at the high end, where our detections lie.

The Schechter function form of the LF used by Dunne et al. (2000) has a very strong exponential roll off at high luminosities. The detection of a small number of very luminous objects, as obtained here, would imply the presence of many more lower luminosity systems. We find three sources in the luminosity range $10^{22.5}-10^{23.5} \mathrm{~W} \mathrm{~m}^{-2} \mathrm{sr}^{-1}$. The Dunne et al. LF would predict that the next lowest dex bin in the LF should contain 100 times more objects. These would have fluxes in the range $0.3-3 \mathrm{mJy}$ at $850 \mu \mathrm{m}$, and would thus be detectable by us either individually or statistically. Yet, we find no evidence for such sources. Perhaps more significantly, sources as luminous as SN1996cf should be extremely rare - so rare in fact that we would not expect to see any at all, given that we are only seeing two sources in the luminosity range $10^{22.5}-10^{23} \mathrm{~W} \mathrm{~m}^{-2} \mathrm{sr}^{-1}$. The assumption of a polynomial tail at high luminosities to the 850- $\mu \mathrm{m} \mathrm{LF}$, rather than an exponential fall off, as suggested for the 60- $\mu \mathrm{m}$ LF (Sanders \& Mirabel 1996) would ameliorate this problem to an extent, but would not provide a complete explanation for the numbers of high-luminosity sources we are seeing, or the absence of sources at somewhat lower luminosities that would still be detectable directly or statistically.

Several explanations are possible.

(i) The LF has evolved from $z=0$ to 0.5 .

At face value, the most obvious explanation for the discrepancies in the luminosity distributions would be evolution in the LF. This would not be one of the standard density or luminosity evolution schemes since this would result in many more moderate flux/luminosity detections than we see. It would also contradict the absence of evolution in the opacity that we find for the sources undetected individually. Instead, some kind of evolution that only affects the high-luminosity objects would be needed, i.e. some kind of luminosity-dependent luminosity or density evolution. This could lead to a bimodal LF.

(ii) The local LF is incorrect as a result of missing objects such as those we have detected.

While the SLUGS is currently the best determination of the local $850-\mu \mathrm{m} \mathrm{LF}$, it consists of less than 200 galaxies. It is thus entirely possible that classes of object are missing from it. If a class of cold dust temperature, high-submillimetre luminosity source existed, they might very well be absent from SLUGS. At the low dust temperatures of our galaxies, $\sim 20 \mathrm{~K}$, such an object at $z \sim 0.1$ would have a $60-\mu \mathrm{m}$ flux $\sim 70 \mathrm{mJy}$, and so would not have appeared in the IRAS catalogues and would not have been targeted by SLUGS or any other submillimetre follow-up. The corresponding 100- $\mu \mathrm{m}$ flux would be $\sim 1 \mathrm{Jy}$, since this is reaching towards the peak of the SED. Such a cold source might be written off as likely cirrus contamination and thus be absent from the main IRAS galaxy catalogues. Its $850-\mu \mathrm{m}$ flux would be about $100 \mathrm{mJy}$, comparable to the $60-\mu \mathrm{m}$ flux. At a redshift of 0.05 , the fluxes would be four times larger, and so such a source would still be only marginally detected by $I R A S$ at $60 \mu \mathrm{m}$. If they exist, such objects would best be detected by large-area, possibly all-sky, shallow surveys with SCUBA2, or in the Planck all-sky surveys.

(iii) The bright targets are not members of the same population as the rest of our targets.

Our application of the low- $z$ LF is based on the assumption that all of our target SN host galaxies come from the same parent population of established galaxies with large stellar populations. This might not be the case. If there was an additional population of SN host galaxies with large submillimetre luminosities not arising from quiescent dust heating, making up $\sim 10$ per cent of SN hosts, then it would not be correct to extrapolate the submillimetre properties of the bulk of the $\mathrm{SN}$ hosts from a few objects coming from a separate population. How might such a situation arise? The obvious class of objects with large submillimetre luminosities are galaxies hosting a substantial burst of star formation, though the low dust temperature in our bright submillimetre objects and their quiescent morphology might argue against this. However, starburst galaxies are not the obvious hosts of Type Ia SNe. They are, though, the likely hosts of other classes of SN. If some fraction of the SN1a's in the surveys are actually misclassified core collapse $\mathrm{SNe}$ in actively star-forming, dusty hosts - they might perhaps be Type Ic SNe, which have masqueraded as Type Ia's in the past (Kotak, private communication; see also Homeier 2005) - then this could provide an explanation. There would, though, be consequences for the SN1a survey results if there is this level of contamination by non-Ia's. Core collapse $\mathrm{SNe}$ are typically of lower luminosity than Type Ia's. Type Ib and Ic SNe, viewed as a class, have absolute magnitudes of about $1.24 \mathrm{mag}$ fainter than Type Ia SNe (Richardson et al. 2002). If there is indeed a $\sim 10$ per cent contamination rate of Type Ia's by Type Ic's 
in the surveys, then this could produce an overall dimming of the mean brightness by $0.24 \mathrm{mag}$. This is comparable to the dimming at $z \sim 0.5$ attributed to the cosmological constant or dark energy term (Reiss et al. 1998; Perlmutter et al. 1999), and thus might have a bearing on the reality or value of this cosmological component. The redshift evolution in the dimming of SN1a's attributed to dark energy at higher redshifts would not easily be mimicked by interloping Type Ic SNe, while the absence of starburst spectral features in these objects is an additional problem for this explanation.

At this stage, it is not possible to choose between these scenarios since the sample of such objects is still very small. Each possible explanation has its own significant implications for astrophysics, so it is important that this strange population is followed up in more detail, and that steps are taken to expand the sample size. At this stage, the only way to find more such objects seems to be observing additional SN1a host galaxies, in which they are represented at $\sim 10$ per cent. This is not a particularly efficient method of discovery. Extension of our SN1a host studies to $z=1$ might prove beneficial, and could test whether these objects are the result of evolution. Aside from that, the best hope for uncovering more of this class might be the cosmological submillimetre surveys being proposed for SCUBA2. Furthermore, a very large area shallow SCUBA2 survey, possibly covering the whole accessible sky, would be one way to test the hypothesis that cold galaxies are a feature of the general galaxy population at all redshifts by looking for their local equivalents. Conversely, detailed study of our existing submillimetre bright targets, in search of signs of recent star formation, might be the best way of testing our third explanation, and thus to remove any lingering doubts about the classification of their SNe. The absence of clear starburst indications or of morphological disturbance in existing data probably makes this final explanation the least favoured of the three we have proposed.

\section{CONCLUSIONS}

We have extended our search for dust in the host galaxies of Type Ia $\mathrm{SNe}$ at $z \sim 0.5$. We combine our new observations with those of Paper 1, and find that there is no evidence for a substantial increase in optical obscuration in the galaxy population between $z=0$ and 0.5. We have, though, detected strong submillimetre emission from one object, in addition to the one strong detection and one weaker detection from Paper 1. HST imaging of the two bright submillimetre sources suggests that they are quiescent, edge-on disc galaxies, in contrast to the disturbed and interacting SMGs, found in blankfield submillimetre surveys and local ULIRGs. We examine the dust properties of these sources, and conclude that they are ultraluminous submillimetre sources, with large dust masses, but with cold dust SEDs, $T \sim 20 \mathrm{~K}$. We also examine the luminosity distribution of the SN1a host galaxies as a class, and compare it to the SLUGS submillimetre LF. We conclude that the $z=0.5$ luminosity distribution seems bimodal, with an absence of sources within a factor of a few of our bright objects. Several scenarios could explain this effect, including some forms of LF evolution, the absence of sources such as these from local submillimetre surveys or problems with the classification of $\sim 10$ per cent of SNe in the Type Ia SN surveys. We propose various ways in which these hypotheses could be tested by future observations.

\section{ACKNOWLEDGMENTS}

The James Clerk Maxwell Telescope (JCMT) is operated by The Joint Astronomy Centre on behalf of the Particle Physics and
Astronomy Research Council of the United Kingdom, the Netherlands Organization for Scientific Research and the National Research Council of Canada. DLC acknowledges funding from PPARC. JA gratefully acknowledges the support from the Science and Technology Foundation (FCT, Portugal) through the research grant POCTI-FNU-43805-2001. The authors would like to thank the staff at the JCMT for their usual excellent support work, and R. Kotak for useful discussions. The research described in this paper was carried out, in part, by the Jet Propulsion Laboratory, California Institute of Technology, and was sponsored by the National Aeronautics and Space Administration. This publication is also based in part on observations made with the NASA/ESA Hubble Space Telescope, obtained from the Data Archive at the Space Telescope Science Institute, which is operated by the Association of Universities for Research in Astronomy, Inc., under NASA contract NAS5-26555.

\section{REF ERENCES}

Alton P., Bianchi S., Rand R. J., Xilouris E. M., Davies J. I., Trewhella M., 1998, ApJ, 507, L125

Blain A. W., 1999, MNRAS, 309, 955

Blain A. W., Smail I., Ivison R. J., Kneib J.-P., 1999, MNRAS, 302, 632

Calzetti D., Heckman T. M., 1999, ApJ, 519, 27

Chapman S. C., Smail I., Ivison R. J., Helou G., Dale D. A., Lagache G., 2003a, ApJ, 573, 66

Chapman S. C., Windhorst R., Odewahn S., Yan H., Conselice C., 2003b, ApJ, 599, 92

Chapman S. C., Blaine A. W., Smail I., Ivison R. J., 2005, ApJ, 622, 772

Clements D. L., Sutherland W. J., McMahon R. G., Saunders W., 1996, MNRAS, 279, 477

Clements D. L. et al., 2004, MNRAS, 351, 447

Dunne L., Eales S. A., 2001, MNRAS, 327, 697

Dunne L., Eales S. A., Edmunds M., Ivison R., Alexander P., Clements D. L., 2000, MNRAS, 315, 115

Eales S. A. et al., 2000, AJ, 120, 2244

Efstathiou A., Rowan-Robinson M., 2003, MNRAS, 343, 322

Farrah D. et al., 2001, MNRAS, 326, 1333

Farrah D., Afonso J., Efstathiou A., Rowan-Robinson M., Fox M., Clements D., 2003, MNRAS, 343, 585

Farrah D., Fox M., Rowan-Robninson M., Clements D., Afonso J., 2004, ApJ, 603, 489 (Paper 1)

Fixsen D. J. et al., 1998, ApJ, 508, 123

Gispert R., Lagache G., Puget J.-L., 2000, A\&A, 360, L1

Holland W. S. et al., 1999, MNRAS, 303, 659

Homeier N. L., 2005, ApJ, 620, 12

Hughes D. H. et al., 1998, Nat, 394, 241

Jenness T., Lightfoot J. F., 2000, SURF - SCUBA User Reduction Facility. Joint Astron. Centre, Hawaii

Kaviani A., Haenelt M. G., Kauffman G., 2003, MNRAS, 340, 739

Mortier J. et al., 2005, MNRAS, in press

Pei Y. C., Fall S. M., Hauser M. G., 1999, ApJ, 522, 604

Perlmutter S. et al., 1999, ApJ, 517, 565

Puget J.-L. et al., 1999, A\&A, 346, 383

Puget J.-L. et al., 1996, A\&A, 308, L5

Reiss A. G. et al., 1998, AJ, 116, 1009

Richardson D., Branch D., Casebeer D., Millard J., Thomas R. C., Baron E., 2002, AJ, 123, 745

Rowan-Robinson M., 2001, ApJ, 549, 745

Rowan-Robinson M., 2003, MNRAS, 344, 13

Sanders D. B., Mirabel I. F., 1996, ARA\&A, 34, 749

Scott S. E. et al., 2002, MNRAS, 331, 817

Smail I., Ivison R. J., Blain A. W., 1997, ApJ, 490, L5 
Smail I., Chapman S. C., Blain A. W., Ivison R. J., 2004, ApJ, 616, 71

Sullivan M. et al., 2003, MNRAS, 340, 1057

Surace J. A., Sanders D. B., Vacca W. D., Veilleux S., Mazzarella J., 1998, ApJ, 492, 116

Tonry J. L. et al., 2003, ApJ, 594, 1
Tresse L., Maddox S. J., 1998, ApJ, 495, 691

Taylor E. L. et al., 2005, MNRAS, 361, 1352

Vlahakis K., Dunne L., Eales S. A. 2005, MNRAS, in press

This paper has been typeset from a $\mathrm{T}_{\mathrm{E}} \mathrm{X} / \mathrm{L} \mathrm{T} \mathrm{E} \mathrm{X}$ file prepared by the author. 